Talía. Revista de estudios teatrales

ISSN-e: 2659-806X

\title{
ASODAT: una plataforma de información sobre el teatro clásico español a partir de bases de datos federadas
}

Teresa Ferrer Valls ${ }^{1}$; Ariadna Fuertes Seder ${ }^{1}$; Raúl Peña Ortiz ; Alejandro García-Reidy²; Lola Josa Martínez ; Héctor Urzáiz Tortajada ${ }^{4}$

Recibido: 16 de junio de 2020 / Aceptado: 19 de abril de 2021

Resumen: Se presenta en este artículo la primera versión en red de ASODAT, una federación de bases de datos sobre el teatro clásico español y su práctica escénica. Se analizan los pasos necesarios para la creación de este sistema federado de bases de datos, sus objetivos y los resultados conseguidos hasta el momento, así como las perspectivas de continuidad que esta plataforma abre para la integración de investigadores y nuevos proyectos. En su estado actual, ASODAT aporta información sobre obras y autores, manuscritos, censuras y canciones relacionados con textos teatrales.

Palabras clave: Teatro español; Siglos XVI y XVII; Bases de datos.

\section{[en] ASODAT: A Platform of Information About Early Modern Spanish Theater Based on a Federation of Databases}

Abstract: In this article we present the first version of the online database ASODAT, a federation of four databases on different aspects of early modern Spanish theater and its performance. We explain the steps that we followed to create this federated system of databases, its objectives, and our preliminary results, as well as the perspectives of continuity and integration that this platform offers to researchers and new projects. In its current state, ASODAT provides information about plays and authors, manuscripts, censorship and songs related to theatrical texts.

Keywords: Spanish theater; Sixteenth and seventeenth centuries; Databases.

Sumario. 1. Introducción. 2. Fase inicial. 3. Estructura. 4. Un ejemplo de uso. 5. Conclusiones. Bibliografía.

Cómo citar: Ferrer Valls, T.; Fuertes Seder, A.; Peña Ortiz, R.; García-Reidy, A; Josa Martínez, L.; Urzáiz Tortajada, H. (2021). ASODAT: una plataforma de información sobre el teatro clásico español a partir de bases de datos federadas, en Talía. Revista de estudios teatrales, 3, 45-57.

\section{Introducción}

Nuestro propósito al plantear este proyecto, hace ya cuatro años, fue aunar los esfuerzos de varios grupos que trabajaban (o querían hacerlo) utilizando como herramienta una base de datos, y que tenían en común que su objeto de investigación era el teatro clásico español, un bien patrimonial al que no solo se pueden adscribir textos dramáticos y autores, sino toda la información documental - muy abundante en el caso de España - que aporta noticias para la reconstrucción de las diferentes prácticas escénicas y su organización e infraestructura. Resulta evidente que la interrelación de los datos ayuda a mejorar el conocimiento de nuestro objeto de estudio y permite visibilizar aspectos que la perspectiva parcial oculta. Nuestra propia experiencia en el tratamiento de los contenidos en algunas de las bases de datos más veteranas, así como el cotejo de las fuentes que nos sirven para llevar a cabo ese tratamiento, pone de manifiesto que la puesta en común de la información hace aflorar contradicciones o vacíos en la documentación, y abre el camino a la posible resolución de problemas, facilitando también estudios de conjunto, tanto a partir de los contenidos de cada una de las bases de datos como de su interrelación. Quizá el caso más llamativo en los últimos años de los resultados a que puede conducir la confrontación de datos haya sido la atribución a Lope de Vega de la autoría de la obra Mujeres y criados, realizada por Alejandro García-Reidy [2013], a partir del cotejo de información reunida en las bases de datos CATCOM y

\footnotetext{
Universitat de València m.teresa.ferrer@uv.es; ariadna.fuertes@uv.es; raul.penya@uv.es

Universidad de Salamanca alreidy@usal.es

Universitat de Barcelona lolajosa@gmail.com

Universidad de Valladolid urzaiz@fyl.uva.es
} 


\section{aSODAT:}

Bases de Datos Integradas del Teatro Clásico Español

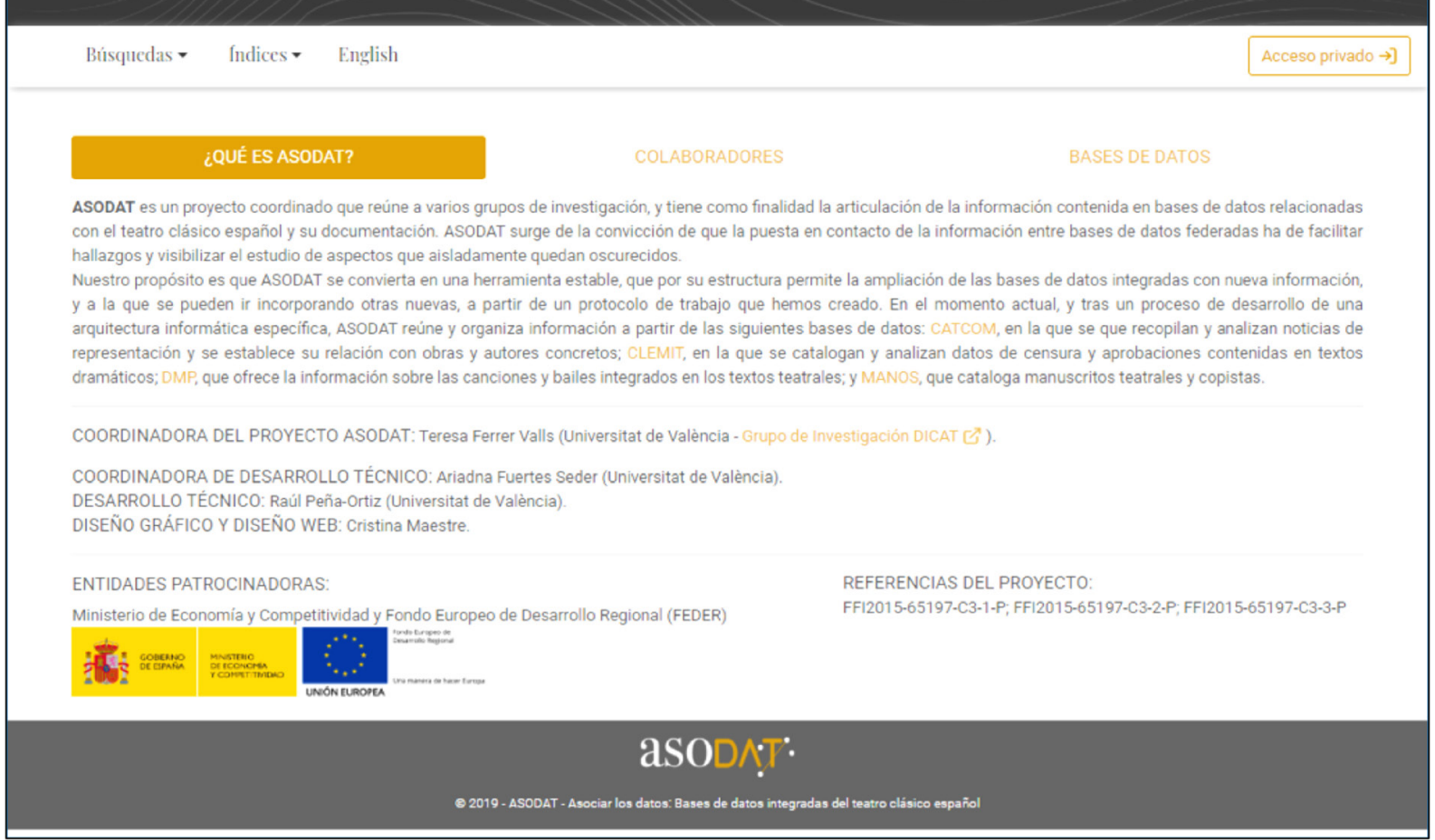

Página de inicio de ASODAT.

MANOS. Pero a este han seguido otros trabajos, y algunas de las revisiones relacionadas con autorías o con estudios sobre los repertorios de algunos dramaturgos que se han llevado a cabo en fechas recientes han surgido de la consulta de algunas de las bases de datos creadas por los grupos que ahora integran esta plataforma de trabajo colaborativo que es ASODAT ${ }^{5}$. La coordinación de grupos de investigación se hizo evidente, pues, desde el comienzo como primer paso para llevar a cabo un proyecto que dotase de un valor añadido a las iniciativas individuales de cada grupo ${ }^{6}$. La coordinación de nuestro trabajo se sostiene sobre los siguientes pilares y parte de la premisa de la importancia del teatro clásico español dentro del patrimonio común europeo:

Por solo mencionar algunos trabajos en relación con el uso que proporcionan las bases de datos para la investigación, pueden verse Ferrer Valls [2013], Fernández Rodríguez [2014], GarcíaReidy [2017, 2019] o Rodríguez Gallego [2017]. También los artículos integrados en el número de la revista Talía coordinado por Urzáiz [2019].

6 Este proyecto está coordinado desde la parte investigadora por Teresa Ferrer Valls, y desde la parte de coordinación técnica y de desarrollo respectivamente por Ariadna Fuertes Seder y Raúl Peña-Ortiz. Ha sido financiado en una primera fase por el Ministerio de Economía y Competitividad y FEDER (referencia FFI2015-6597-C3-1-P). Son IPs de los otros dos subproyectos que han formado parte del coordinado Héctor Urzáiz FFI20156597-C3-2-P y Lola Josa (FFI2015-6597-C3-3). Sobre el planteamiento del proyecto y sus perspectivas iniciales puede verse Ferrer Valls [2018: 115-30].
1. Todas las bases de datos que integran ASODAT se centran en el teatro clásico español y su práctica escénica, desde puntos de vista que resultan complementarios: unas ponen el foco sobre la documentación, otras sobre los textos dramáticos o sobre los aspectos musicales de esos textos.

2. Todos los grupos que conforman este proyecto quieren canalizar su investigación a través del empleo de las herramientas que ofrece el ámbito digital.

3. Partimos de una filosofía basada en que dichos resultados se deben ofrecer en abierto a los estudiosos y, en general, a las personas interesadas en el teatro clásico (directores de escena, compañías, profesores, músicos...).

4. Estamos convencidos de que es necesario salir del ámbito de desarrollo individual de cada proyecto, correlacionando los datos, porque en la medida en que la información crezca y se pueda interrelacionar, la contribución al avance del conocimiento sobre el patrimonio teatral será mayor.

Estos fundamentos son los que nos llevaron a la creación del sistema federado de bases de datos ASODAT (asodat.uv.es) que hemos publicado en diciembre de 2019 como primera versión de un recurso para el estudio del teatro clásico. 


\section{Fase inicial}

Pero para llegar al desarrollo de ASODAT, fue necesario dar unos pasos previos, tanto en cuanto a la conceptualización y estructuración de los contenidos y el establecimiento de una metodología de trabajo común como en cuanto a la estandarización, desde el punto de vista técnico, de las bases de datos preexistentes, un aspecto que trataremos después. Al comienzo contábamos con tres bases de datos (CATCOM, MANOS y CLEMIT), que se caracterizaban por disponer de contenidos generados por trabajos iniciados hace unos años por tres grupos de investigación diferentes, y de una base de datos de nueva concepción (DIGITAL MÚSICA POÉTICA), que fue creada en el marco del proyecto y gestionada por un cuarto grupo. Todas ellas eran y son consultables individualmente y, como se verá por la breve descripción que sigue, todas tienen objetivos que resultan complementarios entre ellos:

- CATCOM (catcom.uv.es) estudia las noticias de representación referidas a títulos de obras dramáticas mencionadas en documentos de procedencia diversa y establece un estado de la cuestión sobre los dramatugos que, en la tradición crítica, se han relacionado con un determinado título. Desde la página de inicio de CATCOM, mediante diferentes pestañas se puede acceder a la consulta de los índices de títulos recogidos hasta hoy en la base de datos y de las fuentes y bibliografía manejadas para el tratamiento de las noticias recopiladas. El formulario de búsqueda incluye un breve índice de instrucciones para la consulta, a través de cada uno de los campos o mediante la combinación de algunos de ellos. El usuario de la base de datos puede realizar indagaciones a partir de títulos, autores, fechas y lugares de representación, directores de compañías, espacios de representación y género (según una tipología general preestablecida). La busca por directores de compañías ofrece un listado que se nutre de los nombres ya registrados en la base de datos DICAT [Ferrer Valls dir. 2008]. La indagación por género se puede llevar a cabo a partir de tres categorías generales ("comedia", para toda obra dramática extensa, "auto", para los autos religiosos, y "pieza breve", en el caso de bailes, jácaras, entremeses...). Esta tipología, al integrarse las bases de datos en ASODAT, se exporta para el resto de bases de datos, evitando la multiplicación del mismo trabajo en cada una de ellas. El rastreo "por espacio dramático" se establece tambien a través de tres categorias generales (palacio/corral/calle). En cuanto a las fechas de representación, el formulario permite obtener un resultado sobre obras representadas en una fecha concreta, dentro del conjunto de registros incluidos en la base de datos, o en un intervalo de fechas $\mathrm{y}$, al ser combinable con otros campos, permite obtener respuestas combinadas (por ejemplo, cuántas obras se representaron en Madrid, en palacio, en un determinado periodo), y a partir de este resul- tado abordar estudios concretos. La base de datos permite también al investigador una indagación a partir de nombres de autores dramáticos, que arroja como resultado el conjunto de obras que se han relacionado con un determinado autor o con varios, según los casos. La problemática que conlleva la atribución de muchas de las obras del acervo teatral del Siglo de Oro explica que en muchas la autoría se desconozca o no se pueda asignar de manera segura y que, a lo largo del tiempo, tanto los catálogos antiguos y recientes como la crítica moderna hayan determinado más de una opción para la atribución. Por lo tanto, nuestro trabajo da cuenta en muchas ocasiones no de una atribución concreta, sino de qué obras se han relacionado con un autor o con más de un autor de manera dudosa, y abre la puerta a la detección por parte de los estudiosos de estos casos contradictorios y su solución. En este aspecto, el de las atribuciones, el proyecto ETSO. Estilometria aplicada al teatro del Siglo de Oro (etso.es), que dirige Germán Vega junto con Álvaro Cuéllar, con los que mantenemos relaciones de colaboración, nos va a permitir avanzar en la clarificación de este panorama.

- La base de datos DIGITAL MÚSICA POÉTICA (digitalmp.uv.es), a partir de ahora DIGITALMP, ofrece información sobre canciones y bailes contenidos en obras teatrales de la época. Desde la página de inicio varias pestañas permiten el acceso al índice de títulos, índice de bibliografía manejada y un índice de primeros versos. Como en la anterior, el formulario incorpora breves indicaciones sobre la utilidad de cada campo, y permite búsquedas por títulos, género (a partir de las categorías generales vistas ya para (ATCOM), nombre de compositor, nombre de poeta/autor, primer verso de una composición y métrica. Además, el campo canción facilita la busca de una palabra contenida en el conjunto de canciones recopiladas en la base de datos (por ejemplo, la palabra "lágrimas" recupera las varias canciones que la contienen). El campo del formulario titulado "tema de canción" da acceso a la selección dentro de una galería de temas genéricos establecidos por el grupo de trabajo y que puede ir aumentando, según las necesidades sobrevenidas que se pueden ir detectando. A partir del análisis de textos teatrales, se extraen datos relativos a las canciones en ellos insertos: localización dentro de la obra, personaje o personajes que intervienen, instrumentos musicales, tipos de estrofas, primeros versos y enlaces con grabaciones de algunas de las piezas. Se ha previsto ir incluyendo, en la medida de lo posible, estos enlaces a grabaciones y la transcripción de algunas partituras en aquellas canciones en las que se han conservado. Pero, como los especialistas saben, las canciones integradas en los textos teatrales de la época no cuentan en su inmensa mayoría con la partitura musical que las pudo acompañar. Como apoyo para quienes quieran reconstruir un posible fondo musical para estas canciones, hemos incluido en los registros de DIGITALMP un apar- 
tado que hemos denominado "sinergias musicales". En él se relacionan, mediante enlaces, canciones de una determinada temática con otras canciones pertenecientes a la misma semántica musical, cuyas partituras se han conservado gracias a otras fuentes, principalmente los cancioneros musicales. Hay que advertir que en muchas ocasiones en la época una tonada musical, bien conocida por el público, era reutilizada o adaptada para una canción con una letra nueva. Hemos establecido enlaces a las composiciones poético-musicales que constan en acceso abierto en la colección "Partituras" de Digital CSIC [digital.csic.es/handle/10261/22377], cuyo contenido hace referencia a las temáticas correspondientes. De esta manera, por ejemplo, ante una hipotética representación en tiempos actuales de cualquier obra analizada y estudiada bajo los parámetros metodológicos de DIGITALMP, puede aplicarse la música de esas piezas afines a aquellas que carecen de música; metódo o procedimiento que denominamos contrafactum y que ya se practicaba en la época. A partir del fondo musical que ofrece DIGITALMP se podrán interrelacionar líneas temáticas musicales conservadas con canciones y bailes cuya música no se ha conservado, abriendo así la posibilidad a la reconstrucción de la sonoridad musical teatral de la época. Además, a partir del tratamiento sistematizado de toda la información reunida, se hará posible constatar, por ejemplo, si existen unos argumentos musicales que corren paralelos a las intervenciones verbales de los personajes que fijan la trama y si, en consecuencia, la música (con su letra y tonadas) establece una relación dialéctica con los mismos, y en qué medida la trama está compuesta por una profundidad o dimensión musical paralela a las acciones de los personajes.

- La base de datos CLEMIT (clemit.uv.es) tiene por objeto de estudio la censura teatral. En su estructura actual, esta base de datos amplía el tratamiento de la información que se ofrecía en la original base de datos llamada "Buscador CLEMIT", ofreciendo un desglose de información que mejora búsquedas y resultados, en un formulario de características similares a los de las dos bases de datos anteriores. Como en CATCOM, existe un pestaña que permite el acceso al conjunto de títulos incluidos en la base de datos, y otra mediante la cual se accede a la bibliografía general empleada. Una tercera incorpora un índice de los nombres de censores registrados hasta este momento. Mediante el formulario se permite la búsqueda por autores, por títulos de obras, por nombres de censores, por fechas de censuras concretas (o por un intervalo de fechas) y por lugares en que se ha llevado a cabo la censura. Cada obra con sus datos principales (autoría, títulos alternativos, fechas de composición, fechas de representación y publicación) se relaciona con los diferentes testimonios conservados de la misma (manuscritos o impresos) y sobre cada uno de ellos se desgranan diferentes informaciones relacionadas con el sistema de censura: licencias de representación obtenidas (lugares y fechas), resultado de la revisión (aprobación, denegación, modificación), intervenciones realizadas sobre el texto de las obras, examinadores y otros agentes que intervinieron en cada censura (cuyos datos biográficos y profesionales también se incluyen). Los resultados obtenidos han de facilitar el análisis desde el punto de vista de su contenido literario, religioso, político, histórico, etc., para que permitan trazar una historia de la censura del antiguo teatro español. Aparte de este tratamiento de la información que ofrece, muchos registros incorporan también un documento en formato PDF, en donde se ofrece una evaluación de esta infomación por parte del investigador responsable del registro de censuras de una obra.

- MANOS (manos.net) está orientada a describir e identificar la manos de los dramaturgos y de los copistas que intervinieron en la elaboración de la gran cantidad de manuscritos teatrales conservados y datados entre finales del siglo XVI y principios del siglo XVIII. El objetivo principal es describir y, en la medida de lo posible, identificar a los copistas que participaron en la ejecución de cada manuscrito, ofreciendo un análisis de las características materiales más significativas del testimonio y trazando la pertenencia de cada manuscrito a compañías profesionales o poseedores privados concretos. El formulario facilita una indagación a partir de personas (autores dramáticos y copistas) y también a apartir de títulos de manuscritos. El resultado de la primera busca ofrece la posibilidad encontrar todos los títulos asociados a la mano de un dramaturgo (en el caso de los autógrafos) o a la mano de un copista. El resultado de la exploración a partir de un título ofrece un registro o una serie de registros que contienen diferentes datos, como la localización del manuscrito, a veces el enlace a la biblioteca que lo custodia, cuando se encuentra digitalizado, y un análisis de sus rasgos gráficos, que justifica la identificación, si es posible, de la mano o manos que han intervenido sobre él. En ocasiones, si el manuscrito contiene nombres de actores, se lleva a cabo la identificación de los mencionados en el manuscrito con los registrados en la base de datos DICAT [Ferrer Valls dir. 2008]. La base de datos cuenta con un registro para cada manuscrito donde se ofrece una muestra de imágenes, una descripción de sus rasgos más notables, la identificación del número de copistas que intervinieron en su elaboración y los rasgos de su caligrafía, e información asociada al uso del testimonio por representantes. La descripción e identificación de cada copista se lleva a cabo a partir de un sistema manual de descripción de letras características, aunque en una próxima etapa se experimentará con métodos automatizados de identificación de caligrafías?

Para esta parte del trabajo, MANOS se beneficia de la financiación del proyecto con referencia PGC2018-096004-A-100 (MCIU/AEI/ FEDER, EU), así como del apoyo económico de la Agencia Estatal de Investigación y el Fondo Social Europeo (Programa Ramón y Cajal 2016 RYC-2016-21174). 
Desde el punto de vista más técnico, en la fase inicial del proyecto se estudió la arquitectura federada como posible opción para el desarrollo de ASODAT, por ser la que proporcionaba un punto intermedio entre la no integración y la integración total de varios sistemas de bases de datos, ya que mantiene la autonomía de cada uno de ellos, pero permite compartir de forma controlada sus datos [Haas y Lin 2002; Hervé 2005; Lindquist et al. 2007; Agüero et al. 2016] $]^{8}$. Esta característica la hace idónea para migrar conjuntos de bases de datos legadas convirtiéndolas en un sistema federado, lo que nos permite también plantearnos de cara al futuro la ampliación con nuevas bases de datos. Como paso previo a la federación se tuvieron que analizar a fondo tanto los contenidos de las bases de datos ya existentes como las propias estructuras y relaciones de los datos, con el objeto de extraer cuáles eran las características deseables para el diseño de la nueva base de datos ASODAT y la información cruzada, procedente de cada una de las bases de datos, que debía contener. Además, se analizaron los diferentes sistemas de gestión de bases de datos (SGBD) sobre los que estaban desarrolladas, llegando a la conclusión de que debían ser estandarizados y homogeneizados, en la medida de lo posible, haciendo que cumpliesen las reglas de normalización de los sistemas de bases de datos relacionales, y que se utilizase software libre para los nuevos desarrollos. Todo esto hizo que se llevasen a cabo las siguientes actuaciones sobre cada una de las bases de datos analizadas:

- La base de datos CATCOM, desarrollada incicialmente en el software propietario FileMaker, fue migrada al SGBD (Sistema Gestor de Base de Datos) MySQL lo que conllevaba la consiguiente normalización y estandarización del sistema. El software de aplicación fue desarrollado en lenguaje de programación PHP con el fin de facilitar la accesibilidad de la información, la homogeneidad y el uso de software libre como parte del sistema final. Hay que señalar, desde el punto de vista de los investigadores humanistas, que esta migración requirió un revisión profunda de testeo de los datos y de funcionamiento correcto de los mismos, así como una adaptación de los investigadores a una nueva metodología de tratamiento e inserción de los datos, en la que debían de confluir los diferentes grupos.

- La base de datos CLEMIT y su aplicación fueron rediseñadas con el fin de facilitar la accesibilidad de la información en el sistema final. Aunque inicialmente se encontraba implementada sobre MySQL había sido desarrollada sin

Un ejemplo de base de datos federadas relacionadas con las Humanidades Digitales ofrece el proyecto RemBench [http://rembench. huygens.knaw.nl/] [Verberne, Boves, y Bosch 2016]. Más cercano a nuestro ámbito, en el caso de la literatura española áurea, la Red Aracne (http://www.red-aracne.es/presentacion) ofrece como solución un metabuscador que abarca todas las bibliotecas digitales que integran esta red y posibilita las búsquedas a partir de metadatos marcados siguiendo el protocolo OAI-PMH. cumplir los requisitos de normalización exigidos para las bases de datos relacionales que son los que garantizan la integridad de la información contenida9 ${ }^{9}$ Así, tras su análisis, tuvo que ser reestructurada por completo con el objeto de sacar el mayor partido de los datos, en su mayoría contenidos en forma de PDF en la vieja base de datos. Este proceso también exigió la inmersión de los investigadores en una nueva metodología para tratar la información que en la antigua CLEMIT se contenía en el PDF, e incorporarla a la estructura de la nueva CLEMIT, que la dota de una mayor productividad gracias a las búsquedas que permite.

- En el caso de la base de datos DIGITALMP, al ser de nueva creación, su diseño desde cero hizo el proceso más sencillo. Atendiendo a los requerimientos de los investigadores y a las necesidades de accesibilidad del sistema final, se creó una estructura de gestión para el trabajo de tratamiento de datos y el SGBD adoptado fue, como en las dos anteriores, MySQL, siendo desarrollado el software de aplicación en PHP.

- Por último, MANOS planteaba una problemática especial, debido a sus particularidades de propiedad intelectual y a la tecnología en la que está desarrollada y desplegada. Desde el punto de vista técnico, el sistema legado está desarrollado en Ruby on Rails (RoR) y como software de gestión de base de datos emplea PostgreSQL. En este caso no se podía mantener la homogeneidad con el resto de sistemas por lo que el modo de federarla debía ser a través del desarrollo de un programa específico de comunicación entre la base de datos MANOS y ASODAT, es decir, un API (Application Programming Interface) y, dado que se iba a trabajar a través de servicios de Internet, se apostó por usar el estandar REST para el intercambio de datos. Por tanto, para facilitar la accesibilidad de la información de MANOS desde el sistema final ASODAT se adoptó un sistema de federación híbrida, es decir, se decidió compartir la información contenida en la base de datos a través de servicios web, creando un API REST a través del cual se comparte la información.

\section{Estructura}

Tras estos pasos previos, se procedió al desarrollo de la arquitectura federada para ASODAT, que se adoptó, en síntesis, por dos motivos principales: 1) Preservar la autonomía de los cuatro grupos de investigación; 2) Facilitar el trabajo colaborativo entre todas las bases de datos mediante la explotación cruzada de la información en el sistema final. En la figura siguiente se muestra la arquitectura de referencia para el sistema de información ASODAT:

\footnotetext{
Los requisitos exigidos para el modelo de una base de datos relacional fueron ya definidos por Codd en un trabajo pionero [1970].
} 


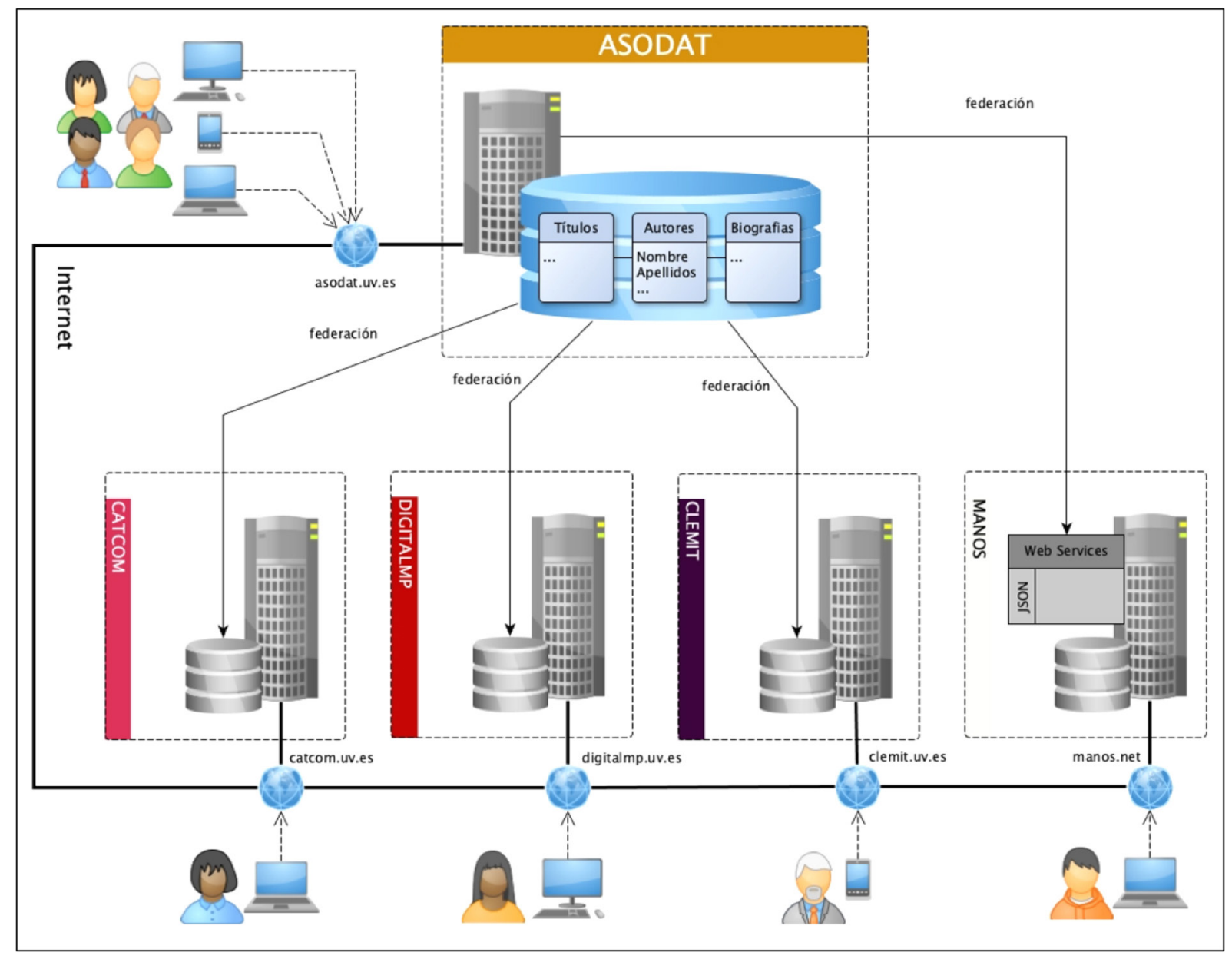

Arquitectura del sistema ASODAT.

Por las razones expuestas anteriormente, y como se refleja en la figura, las fuentes de la federación contempladas en la arquitectura son de dos tipos: fundadas en bases de datos MySQL y en un API REST de servicios web. CATCOM, DIGITALMP y CLEMIT optan por una federación que se sutenta en la compartición de esquema en un sistema de gestión de bases de datos MySQL (MySQL-based federation), el cual ha sido parametrizado a nivel de la aplicación web de ASODAT mediante un patrón de plug-ins (CATCOM Plug-in, DIGITALMP Plug-in y CLEMIT Plug-in) lo que facilita el trabajo frente a la incorporación de nuevas bases de datos MySQL en el futuro. Para gestionar esta reunión de información proviniente de la federación de servidores que contienen nuestras bases de datos se optó por hacer uso del Cluster Galera ${ }^{10}$ dado que permite la federación entre las bases de datos distribuidas MySQL, con las ventajas que esto supone en cuanto a máxima disponibilidad y seguridad de contenidos gracias a sus protocolos de tolerancia a fallos y réplica de bases de datos ${ }^{11}$.

Para conjugar toda la información federada de manera eficiente se adoptó un diseño de federación en dos niveles. En el primer nivel se creó un esquema con-

10 Para información sobre el Cluster Galera, véase https://galeracluster. com/products/

11 Para más detalles técnicos consúltese https://galeracluster.com/wpcontent/uploads/2013/10/Minimizing-downtime-and-maximizingelasticity-with-Galera-Cluster-for-MySQL.pdf ceptual de la información común a todas las fuentes de datos, lo que dio lugar a la propia base de datos común ASODAT. En el segundo nivel se desarrolló un esquema para el cruce de la información de todas las bases de datos y su explotación para la visualización a través de la consulta. Para que se comprenda mejor, vamos a describir dichos niveles, no tanto desde el punto de vista técnico, como en la práctica, a partir de la aplicación diseñada para gestionarlos. En el primer nivel, la aplicación web de ASODAT ofrece dos elementos a partir de los cuales llevar a cabo las búsquedas, elementos comunes a todas las bases de datos y que pertenecen al propio esquema conceptual de la base de datos común ASODAT: los autores y los títulos, cuya integridad y correcto mantenimiento son de gran importancia, dado que son los que sustentan la federación. Por ello, la creación de nuevos autores y de nuevos títulos se gestiona desde ASODAT para todas las bases de datos, por medio de tablas comunes y siguiendo unos protocolos desarrollados con este objetivo, lo que permite corregir errores y establecer vinculaciones entre ellas desde el momento mismo de la creación de un nuevo título o de un nuevo autor, pues la nueva inserción en cada una de las tablas se debe autorizar desde ASODAT. Esto garantiza la uniformidad de menciones de autores y títulos, pero también permite al investigador que solicita una nueva inserción desde la interfaz de gestión, acceder a la información unificada existente en ASODAT y 
cotejar las distintas bases de datos, comprobando así si ya existe información sobre la obra que le interesa y visibilizando la problemática particular que puede ofrecer un título concreto y su relación con variantes de título de una misma obra que puedan encontrarse en otras bases de datos. Este nivel puede ser ampliado en un futuro, según las necesidades y los resultados que quieran perseguir los grupos asociados, sin tener que cambiar la arquitectura del sistema, pues desde ASODAT se gestionan las relaciones entre las distintas bases de datos pertenecientes a la federación. Por otro lado, la asociación evita redundancias de información, $\mathrm{y}$ hace que la atención investigadora de cada grupo se focalice en su objeto de estudio, sin la necesidad de recopilar o marcar datos que ya vienen facilitados por otras bases de datos. Por ejemplo, si la atribución de una obra a un autor (de manera fiable o discutida) viene desarrollada con mayor detalle en CATCOM, se hace innecesario que cada grupo invierta esfuerzos para establecerla. Otro tanto sucede con la categorización por géneros. De este modo, si todos los grupos a la hora de trabajar tienen acceso a través de ASODAT a la información reunida en todas las bases de datos, la discriminación entre obras de títulos idénticos o la decisión sobre la vinculación de una obra desde una base de datos a las contenidas en otras se ve facilitada. Así por ejemplo, un cotejo de la información sobre autoría presente en CATCOM y MANOS permite evitar una identificación errónea entre dos entremeses titulados $E l$ borracho, uno escrito por Luis Quiñones de Benavente y representado por la compañía de Antonio García de Prado, y otro compuesto por Melchor Zapata, del que se conserva una copia manuscrita. Otro ejemplo que también atañe a CATCOM y MANOS es que ambas documentan obras con el título El carretero, pero la primera es una comedia anónima representada en 1617, mientras que la segunda es un baile, por lo que en ASODAT se mantienen registros independientes. En otras palabras, la federación no es una mera suma in- discriminada de datos, pues detrás de cada registro en ASODAT subyace un análisis científico de la información que se cruza por parte de los grupos implicados, y que ya supone un primer resultado que se ofrece al investigador que maneje ASODAT.

En el primer nivel de la federación, pues, la aplicación web de ASODAT ofrece una visión general y permite localizar autores y títulos. El segundo nivel de la federación es el que podemos considerar más dinámico, dado que puede crecer y adaptarse según las necesidades de los investigadores. Es aquí donde la aplicación web facilita el cruce con información más específica proveniente de las bases de datos federadas. En la primera versión del sistema federado, que hemos inaugurado, la información cruzada hace referencia a las noticias de representaciones de las obras (CATCOM), la información literario-musical (DIGITALMP), los testimonios de censura (CLEMIT) o los manuscritos y copistas (MANOS). De este modo los diferentes grupos de investigación pueden seguir trabajando de forma independiente a través de las aplicaciones web desarrolladas específicamente para sus necesidades, mientras que ASODAT ofrece un contexto de colaboración entre los investigadores.

Nos detendremos ahora en el modo en que en estos momentos se ofrece para el usuario externo la consulta de los datos reunidos en ASODAT. El acceso a la consulta se puede hacer a partir de la búsqueda por autores teatrales y de la búsqueda por títulos. Desde el resultado que arroja la consulta de un autor (pongamos por ejemplo, Francisco Agustín de Tárrega), podemos acceder, pulsando en los iconos correspondientes, por un lado a la biografía, y por otro a una pantalla en que se da cuenta de las obras que se atribuyen a este autor, de manera cierta o dudosa, en las diferentes bases de datos, tal y como se muestra en la siguiente figura.

Si pulsamos sobre el icono correspondiente al nombre de dramaturgo, se desplegará un registro con la biografía y la producción dramática completa que se le atribuye, como puede verse en la imagen que sigue más

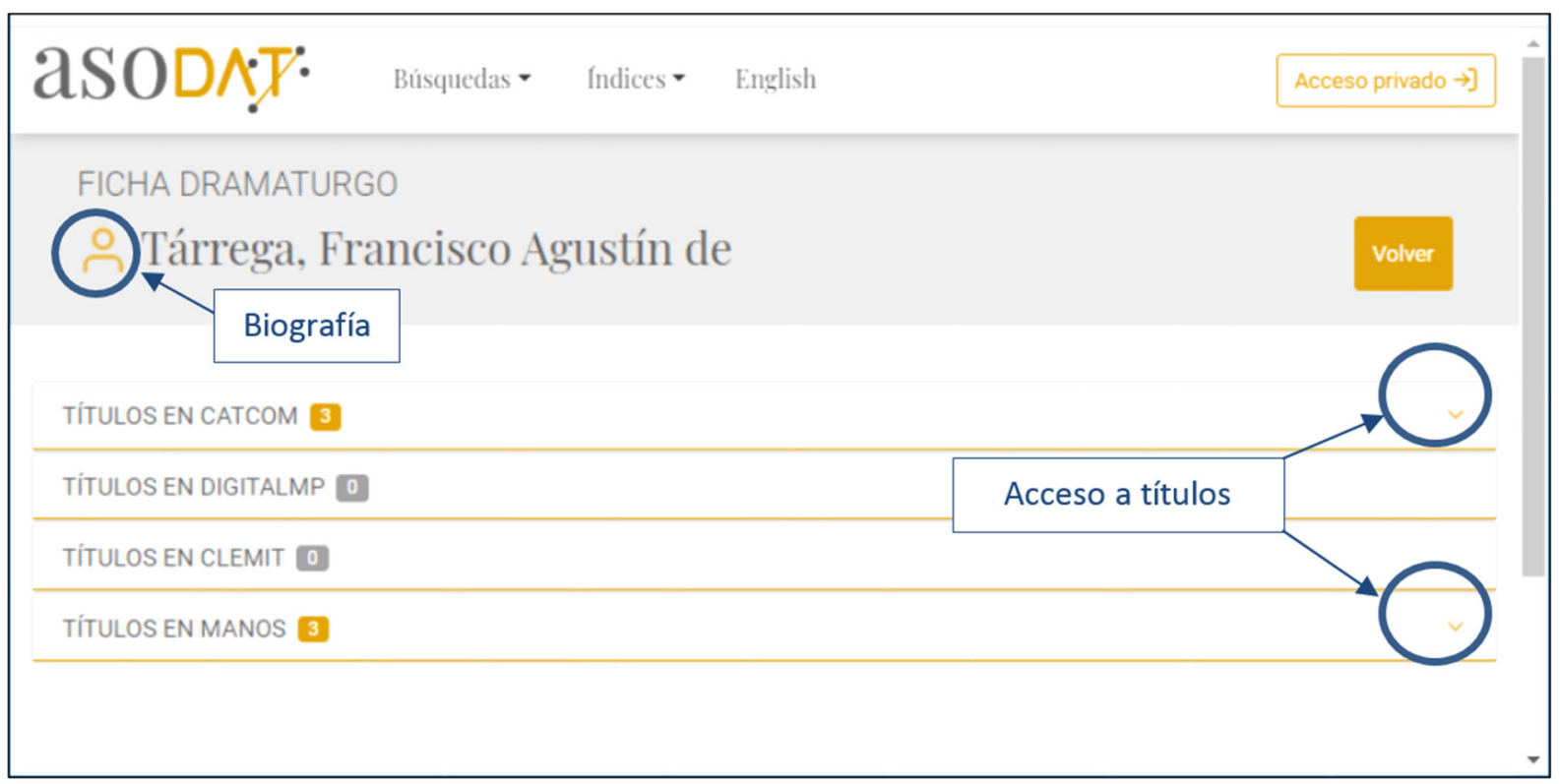

Página de acceso a biografías y títulos. 
abajo. Las biografías que se han incoporado a ASODAT se han implementado a partir del Catálogo de autores teatrales de Héctor Urzáiz (2002), vinculándolas a la tabla de autores. Este recurso resulta un complemento de ayuda propio de la consulta a través de ASODAT, que se pone al servicio de todas las bases de datos (desde la interfaz de gestión), así como de los usuarios externos (desde la interfaz de consulta). Nuestro propósito, ya llevado a cabo sobre unas pocas biografías, como la de Lope de Rueda, es ir actualizándolas progresivamente. Cuando se ha utilizado una bibliografía complementaria a dicho catálogo, esto aparece reflejado en el apartado en donde se recopilan fuente o fuentes bibliográficas empleadas.

Si pulsamos sobre el icono que nos permite el acceso al listado de los títulos asociados a Francisco Agustín de Tárrega procedentes de cada base de datos, veremos, en un rápido vistazo, cuáles son, y pulsando sobre el icono de la derecha de cada título podremos acceder directamente a su contenido completo en la base de datos de origen. El resultado sería el que se ve en la imagen siguiente, si desplegamos los títulos asociados a CATCOM. En este caso, no hay títulos asociados a CLEMIT y DIGITALMP (por eso la cifra del contador marca 0), y hay tres asociados a MANOS, que podríamos ver desplegando mediante el icono de la derecha el listado, como se indica en la figura.

La otra modalidad de búsqueda que actualmente está implementada para el usuario de ASODAT es la búsque- da por títulos que contempla todos los incluidos en las diferentes bases de datos, tanto si se trata de títulos principales como de variantes de un título o segundos títulos de una misma obra.

En el momento de redactar este artículo, ASODAT arroja 3.662 resultados, que se corresponden con títulos de obras, variantes y segundos títulos, como se acaba de indicar. A la hora de poner en marcha ASODAT la vinculación de todos estos títulos y la obras contenidas en las diferentes bases de datos supuso un trabajo muy costoso de revisión de listados de obras y contenidos de cada base de datos para establecer las relaciones existentes entre ellas, que llevaron a cabo varios investigadores de los diferentes grupos implicados. A partir de ahora, con la metodología y el protocolo de trabajo que hemos creado y adoptado por los diferentes grupos, la vinculación se establece desde el momento en que el investigador solicita la inserción de un nuevo título en ASODAT, solicitud que se ha de autorizar tras una revisión de los contenidos de las diferentes bases de datos en las que se encuentre alguno o algunos títulos que, en principio, pudieran ser idénticos o semejantes, para concluir decidiendo sobre el establecimiento de la asociación.

Pondremos un ejemplo del resultado que ofrece una pesquisa por títulos en ASODAT. Si buscamos recabar información desde el formulario de búsqueda sobre una obra como El catalán Serrallonga, ASODAT nos arrojará el siguiente resultado, en el que, como puede verse,

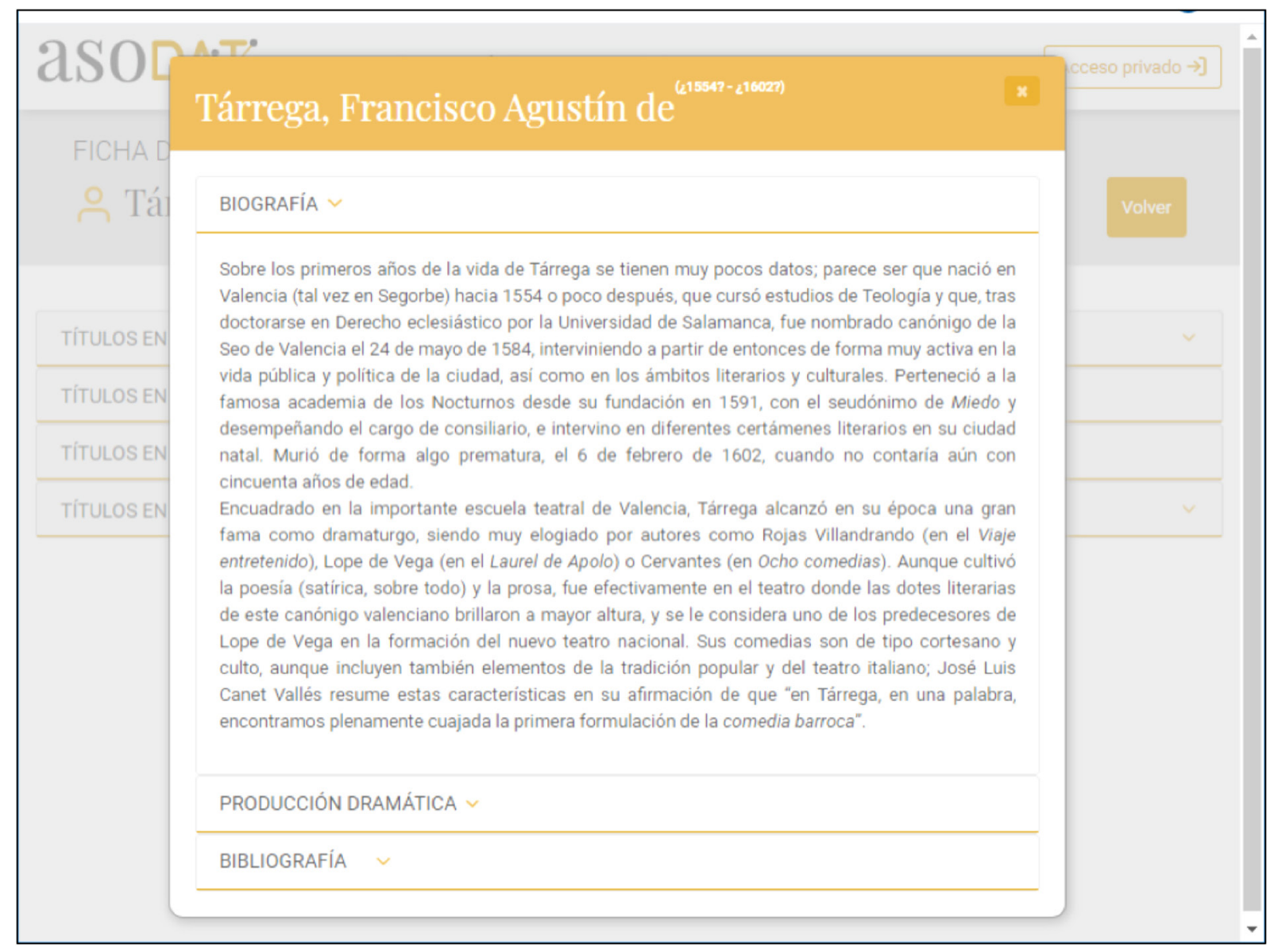

Visualización de la biografía de un dramaturgo. 


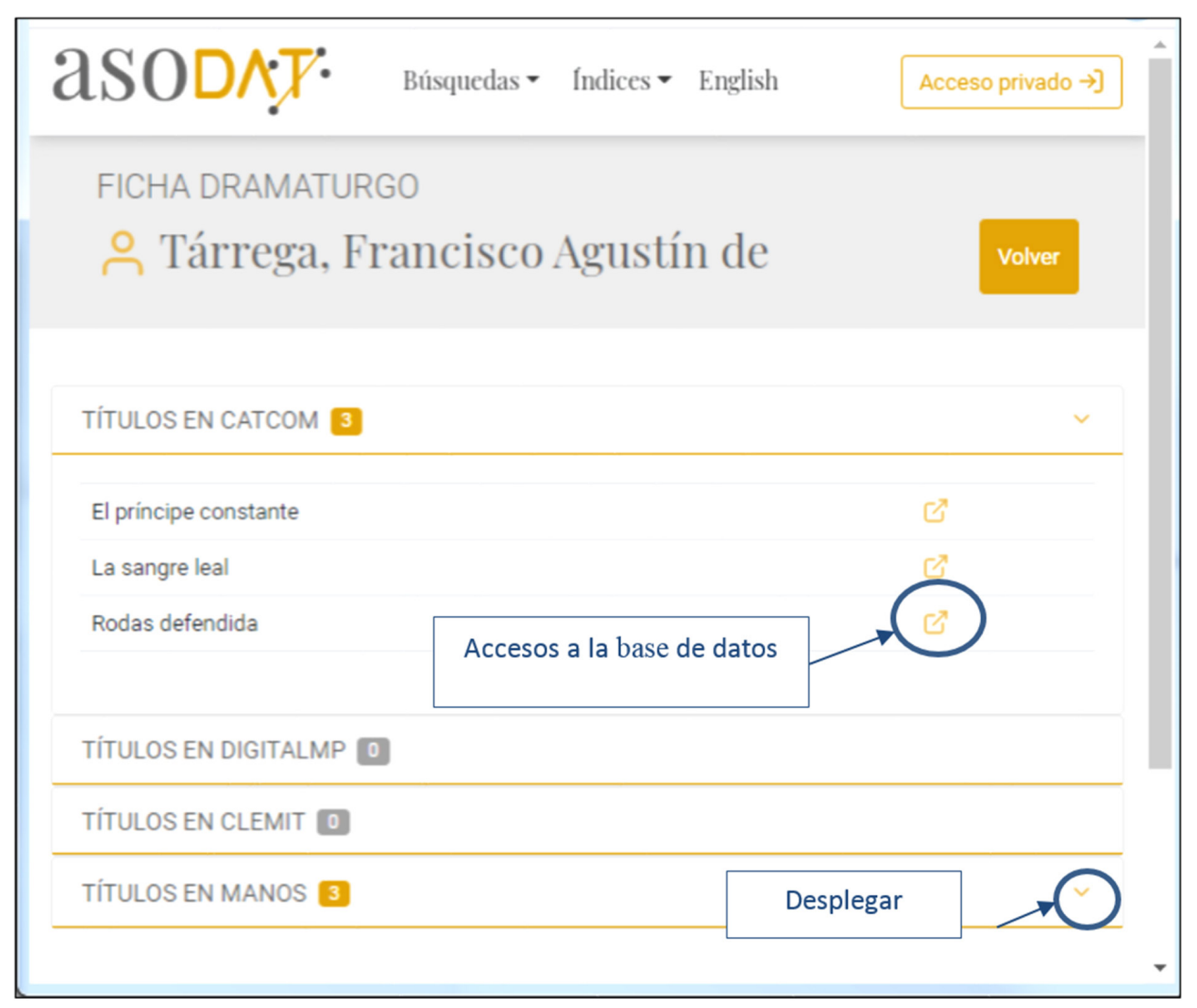

Visualización de títulos relacionados con un dramaturgo.

se indica el título que hemos tecleado en la búsqueda en color negro y en un tono gris algunas de sus variantes o títulos alternativos:

Desde esta pantalla, podremos acceder al registro, en donde se despliegan ya el conjunto variantes y títulos alternativos de esta obra, el género al que se adscribe (según la tipología general que hemos comentado arriba, es decir comedia / auto / pieza breve) y los dramaturgos relacionados con la obra.
En este caso se trata de una obra escrita en colaboración por Francisco de Rojas Zorrilla, Antonio Coello y Luis Vélez de Guevara. Desde esta pantalla se puede acceder a la biografía de cada uno de ellos y a su respectiva producción dramática conocida. El registro ofrece un índice que da cuenta de los diferentes datos recopilados en ASODAT a partir de las distintas bases de datos, y el número de noticias que incluye cada base de datos en cada uno de esos subapartados. Pulsando a la derecha de

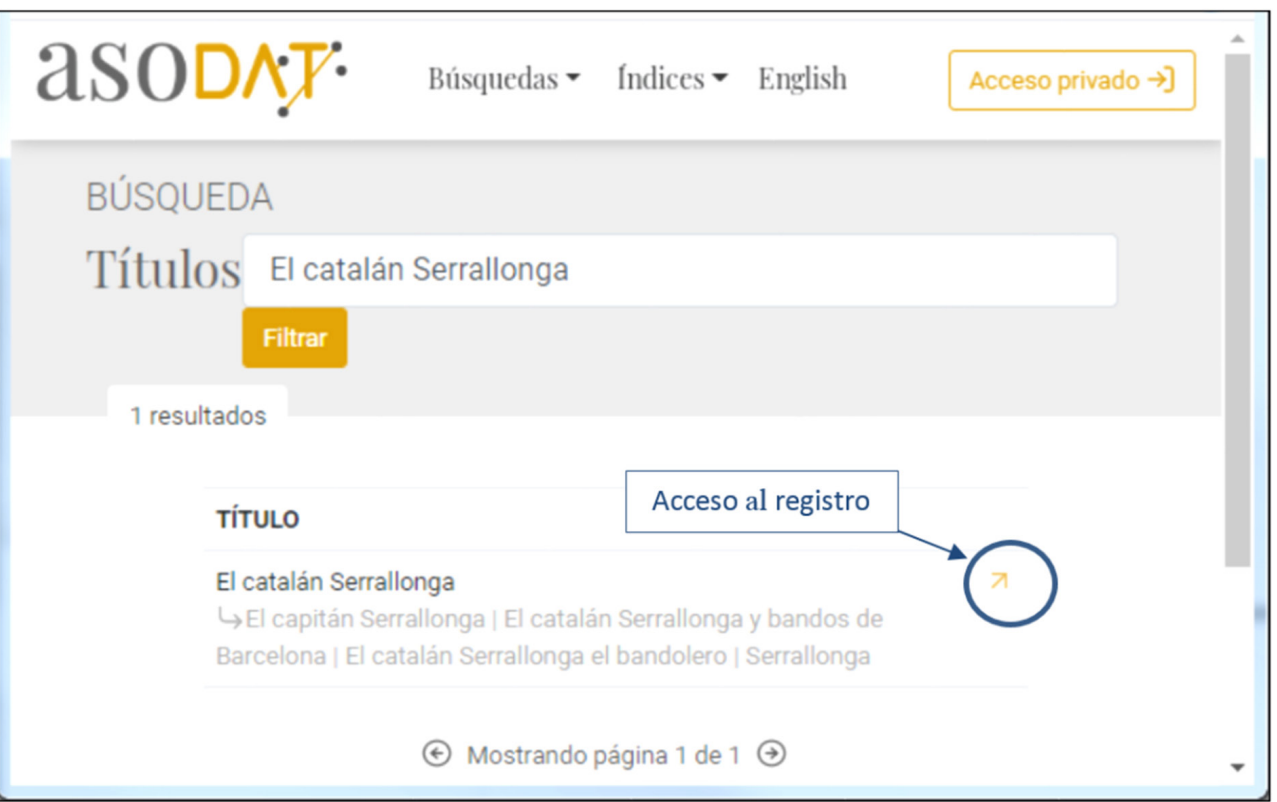

Página de acceso a un título. 


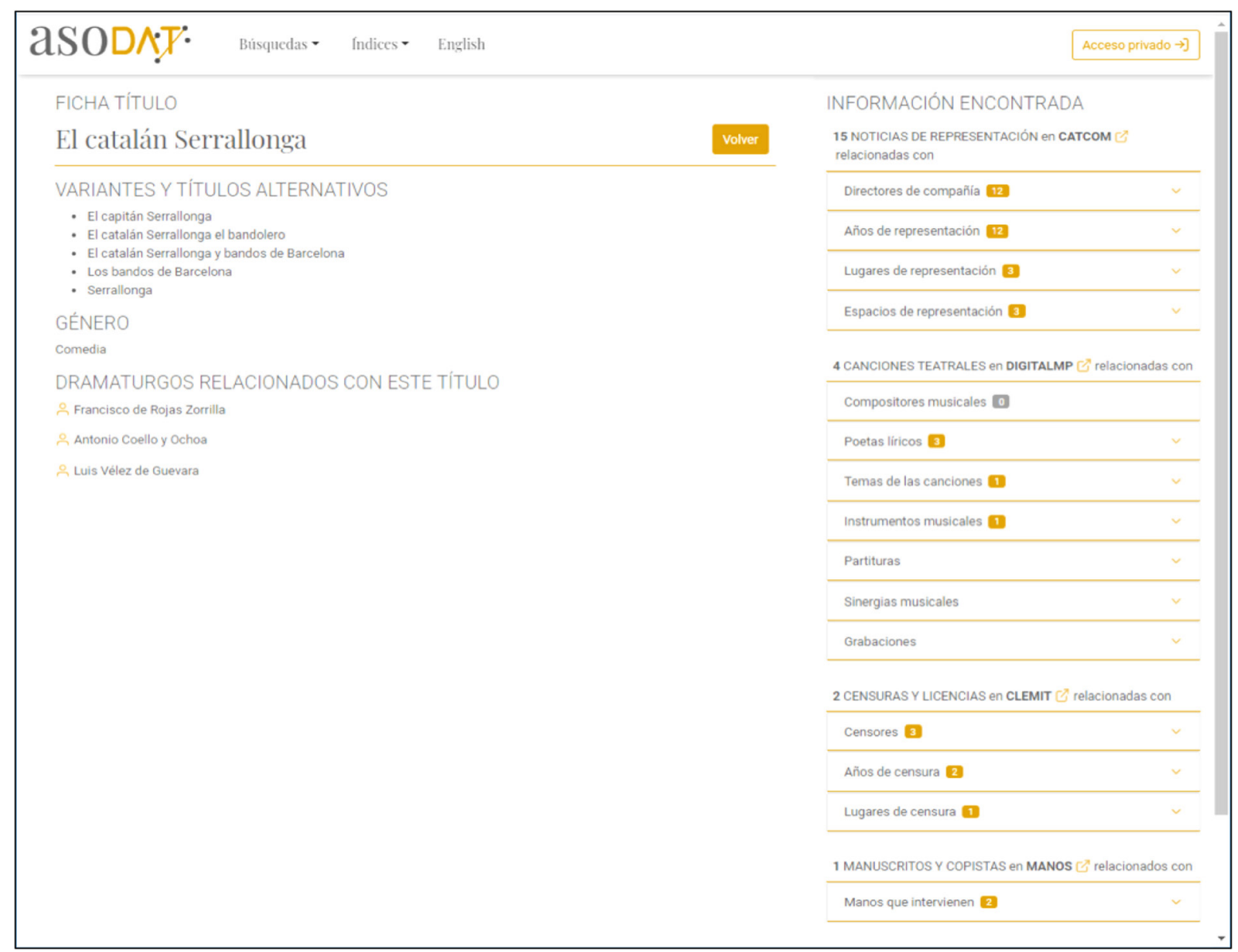

Resultado de la búsqueda por título.

cada uno de ellos, el investigador puede obtener los datos fundamentales que le interesan y acceder también al registro completo, con todas las explicaciones y detalles que ofrece cada base de datos al respecto. Mostraremos un ejemplo del contenido que ofrece, al desplegarse, el subapartado "Años de representación”, que indexa los años en que la obra consta que se representó. Las cifras reflejadas junto a cada año nos informan del número de ocasiones en que hoy sabemos que se representó la obra en un año concreto de los registrados.

\section{Un ejemplo de uso}

ASODAT permite que, a medida que un grupo avance en el trabajo específico de su base de datos, otros grupos puedan beneficiarse rápidamente de esa labor al detectar lagunas o aportar nuevos datos. Por ejemplo, el manuscrito de la comedia Los Silvas y Ayalas, que se conserva en la Biblioteca Nacional de España bajo la signatura Res. 95, es un autógrafo parcial de su autor, el poeta Blas Fernández de Mesa. Al catalogarlo, Paz y Melia [1934] indicó sus características básicas, como la presencia de la firma y rúbrica del autor o el hecho de que el texto de la comedia estaba sacado por más de una mano. El análisis detallado de este testimonio para MANOS apunta una hipótesis de cuál es exactamente la mano de Blas Fernández de Mesa a partir de la comparación con otro manuscrito, pero además revela la existencia, en el vuelto del último folio, de una aprobación para poder representar la comedia, firmada por Pedro de Vargas Machuca en Madrid el 1 de octubre de 1624. Este nuevo dato, obtenido de MANOS, lo hemos podido incorporar a CLEMIT gracias al rápido cotejo de información que ASODAT permite. Además, el trabajo en MANOS a partir de la información que tenemos sobre la actividad de las compañías profesionales en la época, que documenta DICAT [Ferrer Valls dir. 2008], permite plantear la hipótesis de que esta aprobación fue concedida a la compañía dirigida por Juan Martínez de los Ríos, pues es la única formación de representantes que tenemos documentada en Madrid en otoño de 1624. De ser así, la comedia se representó en Madrid entre la fecha de esta aprobación y unos días antes del 6 de noviembre, cuando la compañía de Martínez de los Ríos empezó a representar en Valencia. Por consiguiente, el análisis de este manuscrito en MANOS ha permitido también aportar por deducción una nueva noticia de representación a la base de datos CATCOM.

El hecho de reunir en una misma interfaz información procedente de distintas bases de datos también contribuye a generar nuevas sinergias de investigación al facilitar la detección, de manera rápida y visual, de puntos de contacto entre y áreas de trabajo por explorar. Por ejemplo, tras cotejar aquellos textos que contengan pasajes musicados analizados en DIGITALMP y que 


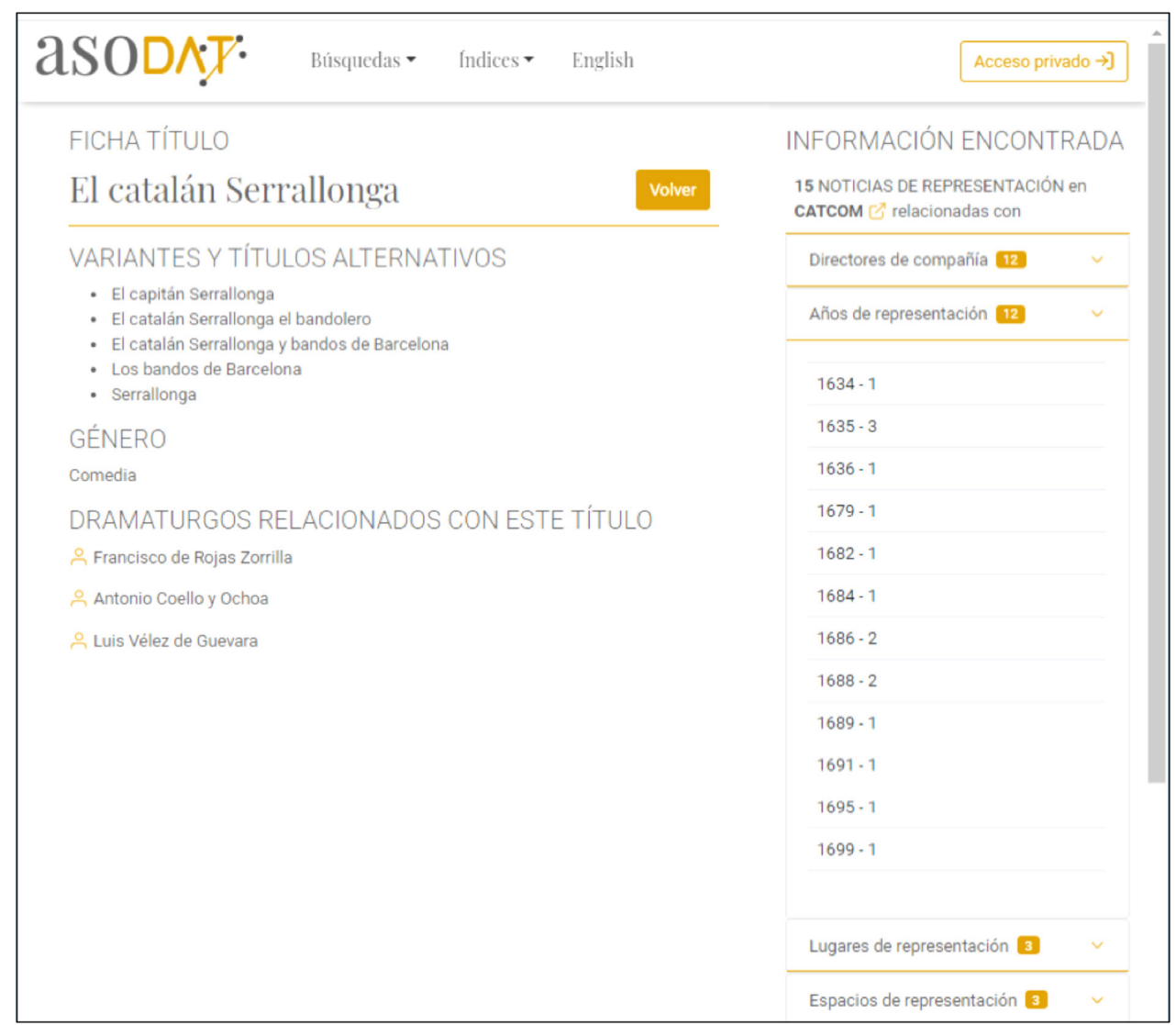

Índice de información y acceso a las distintas bases de datos.

cuenten con testimonios manuscritos, consignados en MANOS, puede ser interesante llevar a cabo estudios específicos acerca de la estabilidad o mutabilidad de pasajes cantados en los manuscritos existentes, o de la detección de patrones que dependan de si los manuscritos se usaron para representaciones cortesanas o representaciones para corral, según la información disponible en CATCOM y CLEMIT. El contraste de la información que permite la federación facilita abordar los datos de manera más manejable, lo que conduce a localizar casos interesantes en los que profundizar y que, de otro modo, podrían pasar desapercibidos entre la enorme cantidad de información que contienen estas bases de datos (y que todavía están creciendo). A modo de ejemplo: si buscamos la palabra "saco" en el buscador de ASODAT, una de las dos comedias con ese término en su título es la obra El saco de la gran casa de Meca, compuesta por Andrés González de Barcia, cuya producción dramática se sitúa entre los años finales del siglo XVII y primer cuarto del siglo XVIII. Se trata de un título llamativo por sí mismo, pero que también funciona como un nodo que conecta la obra con tres de las bases de datos federadas en ASODAT. En la Hispanic Society de Nueva York se conserva un manuscrito autógrafo de esta comedia, en cuya portada figura la fecha de 1695 y la referencia de que la obra se escribió para la compañía dirigida por Carlos Vallejo.

En el primer folio del manuscrito, junto al elenco de personajes, una mano añadió los nombres de los actores y actrices que representarían cada papel. Su análisis en MANOS permite afirmar que formaban parte de la compañía que Carlos Vallejo tenía hacia 1696. Si estos datos se cotejan con la actividad dramática de la formación contenida en DICAT, se puede contextualizar este reparto para una representación que tuvo lugar en Madrid. En el manuscrito figuran también aprobaciones otorgadas en dicha ciudad el 31 de octubre de 1695 por Agustín Gallo Guerrero y Pedro Francisco Lanini Sagredo, quien posiblemente también es responsable de marcar algunos versos de la comedia para eliminar de la representación. De nuevo, se trata de nueva información que se ha incorporado a CATCOM y CLEMIT como resultado de la consulta de ASODAT. Ahora bien, en el registro en MANOS se indica que este manuscrito presenta dos actos segundos y se remite a otro manuscrito de esta misma comedia, conservado en la Biblioteca Nacional de España. Ese segundo manuscrito de El saco de la gran casa de Meca es también autógrafo, aunque parcial, pues le falta el segundo acto, como indicó Paz y Melia [1935]. $\mathrm{Su}$ interés radica no solo en la posibilidad de que uno de los dos segundos actos del manuscrito de Nueva York corresponda al manuscrito de Madrid, sino sobre todo en el hecho de que parece que se tratan de versiones algo distintas de la misma comedia. Así lo sugiere una anotación del siglo XVIII que encontramos en el vuelto del primer folio: "es una misma comedia aunque varía en algo de la otra, y esta aqu[í] más viva y perceptible la idea de el autor. Cotéjense con la impresa". Tenemos, pues, un caso de posibles versiones de autor que no se ha estudiado hasta la fecha. La referencia de la anotación a la existencia de una versión 


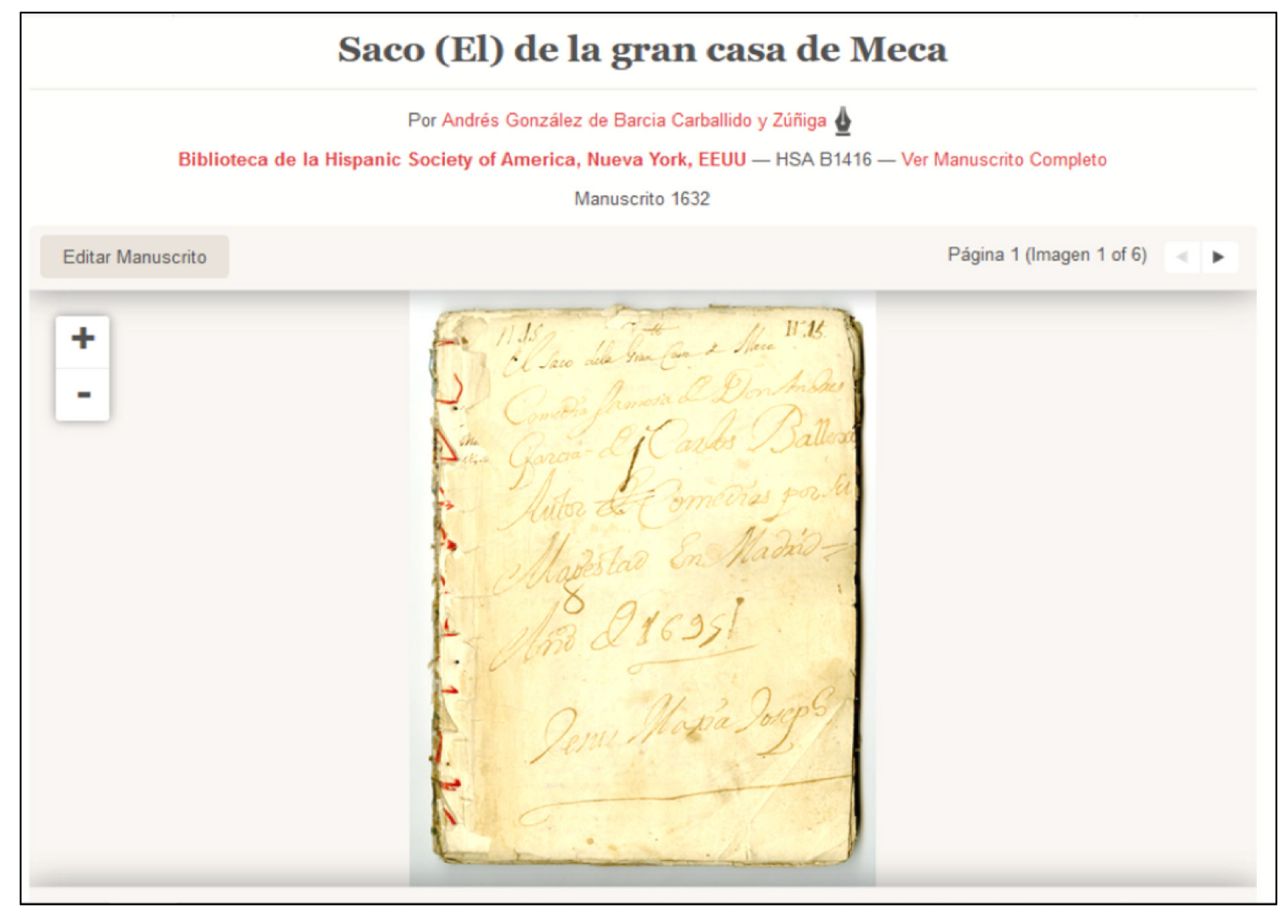

Imagen de un manuscrito en MANOS.

impresa también añade un elemento nuevo, pues una somera búsqueda bibliográfica no reporta resultados sobre la existencia de una edición de esta comedia. Si sumamos a esto el hecho de que Andrés González de Barcia escribió sus comedias bajo diversos pseudónimos, nos encontramos ante un texto y un dramaturgo de entresiglos que pueden merecer más atención crítica por parte de los investigadores.

\section{Conclusiones}

En conclusión, el enfoque de arquitectura que hemos elegido nos permite tener un sistema con perspectiva de futuro, de cara a la continuidad del proyecto, pues resulta, por un lado, flexible, en el sentido de que podemos en etapas sucesivas integrar nuevas bases de datos que cumplan unos requisitos mínimos de estructura y, por otro lado, adaptable a las necesidades de los investigadores, dado que se pueden seguir implementando nuevas relaciones entre las bases de datos desde el nivel superior de la federación. Aquellas que no puedan ser integradas dentro de la federación como una base de datos MySQL, por motivos tecnológicos o de propiedad intelectual, podrán ser incorporadas a la federación a través de la implementación de un plug-in (API-based federation) que permita a ASODAT extraer la información de interés de manera controlada invocando un servicio web en la base de datos objeto de la integración, como se ha hecho en el caso de MANOS. Creemos que nuestros protocolos de trabajo y nuestra experiencia en el ámbito del desarrollo técnico pueden ser beneficiosos para el surgimiento de nuevos grupos y nuevas herramientas integradas, o para la transformación de otras de cara a la federación. Nuestro propósito, como anunciamos al comienzo, es integrar grupos y bases de datos nuevas (que quieran sumarse a esta iniciativa), o facilitar el diálogo con otras que son de referencia en el ámbito del teatro clásico como EMOTHE (emothe.uv.es), ARTELOPE (artelope.uv.es) o DICAT [Ferrer Valls dir. 2008] ${ }^{12}$. Un tipo de plataforma como la que planteamos ha de permitir que unos nos aprovechemos del trabajo de los otros y que el resultado no sea una imagen fija, sino cambiante, en función de nuevas necesidades y de la nuevas preguntas que se puedan formular como consecuencia de su uso o de nuevas situaciones sobrevenidas por la incorporación de otros grupos y proyectos, que sirvan para ampliar la red, pero también nos obliguen a repensarla desde el punto de vista conceptual humanístico y técnico. Esto abre otra reflexión sobre la conservación de este enorme trabajo de años, que no es un objeto digital fijo, y que no requiere tan solo de una biblioteca digital, de un lugar de almacenamiento, sino de instituciones con un personal especializado en el mantenimiento y conservación de este patrimonio. Pero eso es otra cuestión que abre un debate del que no debemos participar solo los investigadores, sino también las instituciones encargadas de la conservación de nuestro patrimonio humanístico, que deberán adaptarse a las necesidades generadas por las nuevas tecnologías.

12 Sobre las posibilidades que abre la asociación de DICAT a la federación, se incluye un artículo en este mismo número de Talía, a cargo de Josefa Badía Herrera, investigadora del grupo DICAT. Ya en prensa el presente trabajo, nuestro proyecto ha recibido nueva financiación del Ministerio de Ciencia e Innovación y FEDER (ref. PID2019-104045GB-C51, PID2019-104045GB-C52, PID2019104045GB-C53). En esta segunda fase se integran en el equipo coordinado dos subproyectos más, el dirigido por Jesús Tronch Pérez, que gestiona las bases de datos, ya en marcha EMOTHE, y ARTELOPE (PID2019-104045GB-C54), esta última en colaboración con Joan Oleza, y el dirigido por Alejandra Ulla Lorenzo (PID2019$104045 \mathrm{~GB}-\mathrm{C} 55)$, con cuyo grupo se va a desarrollar la base de datos ISTAE (Impresos sueltos del teatro antiguo español) para su federación en ASODAT. 


\section{Bibliografía}

Agüero Torales, Marvin Matías, Mario Rubén Canto, y José Luis Vázquez Noguera, (2016): “Sistemas de Bases de Datos Federadas para la Gestión de la Información”. Recurso web <https://doi.org/10.13140/RG.2.2.29045.88802>. Fecha de consulta: 19 de marzo de 2020.

Codd, Edgar Frank (1970): “A Relational Model of Data for Large SharedData Banks", Communications of the ACM, 16-6: 77-387. <https://doi.org/10.1145/362384.362685>.

Fernández Rodríguez, Daniel (2014): "Nuevos datos acerca de los repertorios teatrales en el primer catálogo de El peregrino en su patria", Studia Aurea, 8: 277-314.

Ferrer Valls, Teresa (dir.), Josefa Badía Herrera, Alejandro García Reidy et al. (2008). Diccionario biográfico de actores del teatro clásico español. DICAT, Kassel, Edition Reichenberger.

Ferrer Valls, Teresa (2013): "Lope representado en palacio en la época de Felipe IV: hipótesis a partir de la base de datos CATCOM", Teapal. Teatro de palabras. Revista sobre teatro áureo, 7: 173-192.

Ferrer Valls, Teresa (2018): "Hacia un sistema integrado de bases de datos sobre teatro clásico español y práctica escénica: el proyecto coordinado ASODAT”, Cuadernos AISPI. Estudios de lenguas y literaturas hispánicas, 11: 115-30.

García-Reidy, Alejandro (2013): "Mujeres y criados, una comedia recuperada de Lope de Vega", Revista de Literatura, LXXV.150: 417-438.

García-Reidy, Alejandro (2017): "Las comedias de Calderón en la escena barroca: posibilidades y límites a partir de la base de datos CATCOM", Criticón, 130: 93-107.

García-Reidy, Alejandro (2019): "La presencia escénica barroca de Andrés de Claramonte a partir de las bases de datos Manos y CATCOM", Bulletin of the comediantes, 71. 1-2: 135-54.

Haas, Laura y Eileen Lin (2002): "IBM Federated Database Technology" (White Paper), IBM Corporation. Recurso web $<$ https://www.ibm.com/developerworks/data/library/techarticle/0203haas/0203haas.html $>$. Fecha de consulta: 3 de marzo de 2020.

Lambea, Mariano y Lola Josa (en curso). Partituras. Colección de Digital CSIC-Institució Milá i Fontanals. Recurso web $<$ https://digital.csic.es/handle/10261/22377>. Fecha de consulta: 19 de marzo de 2020.

Lejeune, Hervé (2005): Technical comparison of Oracle vs. SQL Server 2000: Focus on performance. Contributing reviewers: Vineet Buch, Sandra Cheevers, Rick Greenwald, Oracle Corporation. Recurso web <https://www.oracle. com/technetwork/database/performance/twp-perf-oracle.pdf $>$. Fecha de consulta: 3 de marzo de 2020.

Lindquist, David B., Hari Madduri, C. J. Paul y B. Rajaraman (2007): "IBM service management architecture", IBM Systems Journal, 46, 3. Recurso web < https://doi.org/10.1147/sj.463.0423>. Fecha de consulta: 3 de marzo de 2020.

Paz y Melia, Antonio (1934): Catálogo de las piezas de teatro que se conservan en el Departamento de Manuscritos de la Biblioteca Nacional. $2^{\mathrm{a}}$ edición aumentada, Madrid, Patronato de la Biblioteca Nacional, 3 vols.

Rodríguez Gallego, Fernando (2017): “La tentación de lo serio: a propósito de El astrólogo fingido", Anuario Calderoniano, 10: 219-36.

Urzáiz, Héctor (2002): Catálogo de autores teatrales del siglo XVII, Madrid, FUE. 2 vols.

Urzáiz, Héctor, coordinador (2019): “La censura del teatro clásico español”, Talía. Revista de Estudios Teatrales, 1: 1-8.

Verberne, Suzan, Boves, Lou, y Bosch, Antal van den (2016): "Information access in the art history domain: Evaluating a federated search engine for Rembrandt research", Digital Humanities Quarterly, 10, 4. Recurso web <https://dblp. org/db/journals/dhq/dhq10.html>. Fecha de consulta: 17 de marzo de 2020. 